\title{
(2) OPEN ACCESS \\ Uterine fibroids and incidence of depression, anxiety and self-directed violence: a cohort study
}

\author{
Stephanie E Chiuve (1) , ${ }^{1}$ Carrie Huisingh, ${ }^{1}$ Natalia Petruski-Ivleva, ${ }^{2}$ Charlotte Owens, ${ }^{1}$ \\ Wendy Kuohung, ${ }^{3}$ Lauren A Wise ${ }^{4}{ }^{4}$
}

\begin{abstract}
- Additional supplemental material is published online only. To view, please visit the journal online (http://dx doi.org/10.1136/jech-2020214565).
\end{abstract}

${ }^{1}$ AbbVie, North Chicago, Illinois, USA

${ }^{2}$ Science Department, Aetion, New York, New York, USA ${ }^{3}$ Department of Obstetrics and Gynecology, Boston University School of Medicine, Boston,

Massachusetts, USA ${ }^{4}$ Department of Epidemiology, Boston University School of Public Health, Boston, Massachusetts, USA

Correspondence to Dr Stephanie E Chiuve, AbbVie, North Chicago, IL 60064, USA stephanie.chiuve@abbvie.com

Presented at the

International Conference on Pharmacoepidemiology and Therapeutic Risk Management, 24-28 August 2019,

Philadelphia, PA, USA

Received 11 May 2020 Accepted 20 June 2021 Published Online First 22 July 2021

\begin{abstract}
Background Depression and anxiety are prevalent among women with uterine fibroids (UF). The rate of mental health diagnoses in women with UF has not been studied.
\end{abstract}

Methods Women aged $18-50$ years with diagnosed UF were identified in the Optum Clinformatics commercial insurance claims database (Optumlnsight, Eden Prairie, Minnesota) from 1 May 2000 to 31 March 2020 $(n=313754)$ and were matched 1:2 on age and calendar time to women without ( $n=627539$ ). Cox proportional hazards models estimated HRs and 95\% Cls between UF and diagnosed depression, anxiety and self-directed violence, adjusting for demographics and comorbidities. Among women with diagnosed UF, the association between hysterectomy and mental health outcomes was estimated.

Results After adjusting for confounders, women with diagnosed UF had a higher rate of depression (HR: 1.12; $95 \% \mathrm{Cl} 1.10$ to 1.13 ), anxiety (HR: $1.12 ; 95 \% \mathrm{Cl} 1.10$ to 1.13 ) and self-directed violence (HR: $1.46 ; 95 \% \mathrm{Cl}$ 1.29 to 1.64$)$ than women without. Among women with pain symptoms and heavy menstrual bleeding, the HR comparing women with diagnosed UF to women without was $1.21(95 \% \mathrm{Cl} 1.18$ to 1.25$)$ for depression, 1.18 $(95 \% \mathrm{Cl} 1.15$ to 1.21$)$ for anxiety and $1.68(95 \% \mathrm{Cl}$ 1.35 to 2.09) for self-directed violence. Among women with diagnosed UF, the HR comparing women who underwent a hysterectomy to women who did not was $1.22(95 \% \mathrm{Cl} 1.17$ to 1.27$)$ for depression, $1.13(95 \% \mathrm{Cl}$ 1.09 to 1.17$)$ for anxiety and $1.86(95 \% \mathrm{Cl} 1.39$ to 2.49$)$ for self-directed violence.

Conclusions Rates of depression, anxiety and selfdirected violence were higher among women with diagnosed UF, particularly among those who experienced pain symptoms or who underwent hysterectomy.

\section{INTRODUCTION}

Uterine fibroids (UF) are the most common benign gynaecological tumours in women and the primary indication for hysterectomy in the USA. ${ }^{1}$ The lifetime risk of ultrasound-detected UF is $80 \%$ in Black women and 70\% in White women, ${ }^{2}$ and 20\%-50\% of women with UF experience associated symptoms that may significantly impact their quality of life. ${ }^{3}$

The prevalence of depression and anxiety is high among women with $\mathrm{UF}^{4}$ and depressive symptoms and diagnosed depression have been associated with a greater risk of UF. ${ }^{5}$ The risk of depression and anxiety is higher among women with gynaecological conditions such as endometriosis ${ }^{6}$ and polycystic ovary syndrome, ${ }^{7}$ relative to women without these conditions. However, studies evaluating the risk of incident mental health conditions in women with UF are limited. ${ }^{8}$ Important gaps remain in understanding the clinical presentation of other mental health conditions such as self-directed violence, and the extent to which this presentation is modified by age, race/ethnicity or symptoms of UF.

We compared the incidence of diagnosed depression, anxiety and self-directed violence among women with and without diagnosed UF. We further assessed the heterogeneity of these associations by demographics, including UF symptom-based phenotypes and hysterectomy procedures.

\section{MATERIALS AND METHODS \\ Data source}

We conducted a cohort study within the Optum Clinformatics DataMart database that includes healthcare utilisation data for a large nationwide commercial insurer, during 1 May 2000 to 31 March 2020. The database contains transactional data on reimbursement for outpatient prescription dispensing and inpatient and outpatient services, including diagnoses and procedures for $\sim 86.3$ million individuals. All study data were accessed using techniques compliant with the Health Insurance Portability and Accountability Act.

\section{Exposure ascertainment}

The base study population included women aged 18-50 years who were continuously enrolled in the health plan at least 183 days prior to and on the date of cohort entry. A 45-day gap in coverage was allowed. Women were excluded if they had evidence of a mental health condition (medical claim or a prescription claim for a filled antidepressant or antianxiety medication), prevalent cancer (to reduce potential for detection bias) or a hysterectomy (to reduce confounding by hysterectomy) during the baseline period. The baseline period included all available data prior to or on cohort entry date.

Women were included in the UF cohort if they met the following criteria: (1) $\geq 2$ inpatient or outpatient claims $\geq 1$ day apart with UF International Classification of Diseases Ninth Revision (ICD-9) and ICD-10 diagnosis codes in any position, or (2) one inpatient or outpatient claim with UF diagnosis code preceded by ultrasonography or pelvic MRI within 30 days prior ('image confirmed' UF) (online supplemental table 1). The cohort entry 
date was the date of the second claim or the date of the UF claim following imaging.

Women with UF were categorised into one of four symptombased phenotypes based on the presence of symptoms during baseline. The four mutually exclusive groups were (1) heavy menstrual bleeding (HMB) only, (2) pain only, (3) HMB and pain, and (4) neither HMB nor pain. The pain phenotype included women with ICD diagnosis codes for dysmenorrhoea, dyspareunia or pelvic pain. The HMB phenotype included women with ICD diagnosis codes for HMB (menorrhagia or menometrorrhagia), anaemia or blood transfusion.

The unexposed comparator group was sampled from women in the base population without a diagnosis of UF but who had a claim for a general medical or annual gynaecological examination on the same date as the cohort entry date for the matched exposed patient using risk set sampling. For each woman with diagnosed UF, up to two women from the referent cohort were matched on age ( \pm 1 year) and cohort entry date. Women who had a myomectomy on or before cohort entry were excluded from the referent population to minimise exposure misclassification; women with a prior myomectomy were eligible to be in the exposure group if they met the criteria for UF. Women with prior uterine artery embolisation (UAE) procedures remained in the unexposed group, as these procedures can be used to reduce bleeding from causes other than UF.

\section{Outcome ascertainment}

Diagnosed depression and anxiety were identified using ICD-9 and ICD-10 diagnosis codes ${ }^{9}$ and self-directed violence was defined using ICD-9 and ICD-10 codes for a range of violent behaviours including suicidal behaviour, intentional self-inflicted injury or self-harm and suicide ideation. ${ }^{10}$ Postpartum depression was not included as a depression outcome. In sensitivity analysis, outcomes of depression and anxiety were defined using both diagnosis codes and prescription claims. For self-directed violence, codes for suicidal behaviour and intentional selfinflicted injury or self-harm were examined separately because they identify injuries resulting from intentional self-harm ${ }^{10}$ and are indicators of extreme psychological distress which may require more extensive evaluation or intervention, ${ }^{11}$ whereas suicide ideation can be a risk factor for subsequent suicidal behaviours but does not focus on the actual suicide process.

\section{Other covariates}

ICD-9 and ICD-10 diagnosis codes and published validated algorithms used to define all variables in the baseline period are listed in online supplemental table 1. Race/ethnicity data were collected from a combination of public records (eg, driver's licence records, voter registration data) and consumer data (eg, surveys, purchase transactions), where available. Approximately $70 \%$ of these data were imputed using commercial software (E-Tech by Ethnic Technologies). ${ }^{12}$ National Drug Code information was used to detect fills for prescription medications, which served as a proxy for medication use during the baseline period.

\section{Statistical analysis}

Follow-up time began 1 day after the cohort entry date (ie, UF diagnosis) until the occurrence of the event (depression, anxiety or self-harm), database disenrolment, death or end of data collection (31 March 2020), whichever occurred first. Women in the referent group with a diagnosis of UF during the follow-up period were censored on the date of diagnosis ( $7 \%$ of women). Cox proportional hazards regression models estimated HRs and
95\% CIs for the association between UF and each outcome, matched on age and calendar time. Multivariable models were further adjusted for potential confounders identified by the literature and an assessment of a casual diagram. We included variables that were independent risk factors for mental health outcomes. We did not adjust for potential downstream effects of UF (ie, symptoms, infertility) as these factors may be intermediary variables in the causal pathway. We stratified associations according to age $(<30,30-39, \geq 40)$, race and by decade (2000-2010 and 2011-2020) to account for changes in clinical practice over time.

\section{Secondary analysis}

In a secondary analysis restricted to women with diagnosed UF, we compared rates of diagnosed mental health conditions in women who underwent a hysterectomy after UF diagnosis (exposed group) to women who did not (referent group). The cohort entry date for the exposed group was date of hysterectomy. The referent group included women matched 1:2 on age ( \pm 1 year) and cohort entry date who had not undergone a hysterectomy on or before the date of hysterectomy of the exposed patient (figure 1). We excluded women who had a diagnosis of depression, anxiety, self-directed violence, cancer or a prescription claim for a filled antidepressant or antianxiety before the cohort entry date. Multivariable Cox proportional hazards regression models, matched on age and calendar time, estimated HRs and 95\% CIs for each outcome in women with UF who underwent a hysterectomy during follow-up and women who did not. These models were further adjusted for other UF treatments (myomectomy or UAE) prior to hysterectomy. Women in the referent category were censored on the date of a hysterectomy that occurred after the matching index date (9.7\% of women).

All analyses were performed using Aetion Evidence Platform V.4.17. P values were two tailed, and significance was set at $\mathrm{p}<0.05$.

\section{RESULTS}

Of those eligible, 313754 women with UF were matched to 627 539 women without UF (figure 1). Median follow-up time was 596 days (IQR: 231, 1293) for anxiety, 608 days (IQR: 236, 1319) for depression and 665 days (IQR: 258, 1445) for selfdirected violence. The most common symptoms among women with UF were pelvic pain and HMB (table 1). Women with diagnosed UF who were excluded due to diagnosis of mental health conditions had a greater frequency of symptoms, comorbidities and medication use compared with women included in the study (online supplemental table 2). During follow-up, over half of the depression and anxiety claims came from a primary care or mental health provider and approximately $30 \%$ of self-directed violence events were captured by claims from emergency medicine or primary care providers (online supplemental table 3 ).

After adjusting for potential confounders, diagnosed UF was associated with higher rates of diagnosed depression, anxiety and self-directed violence (table 2). When the referent group was not censored on UF diagnosis, the results were not appreciably altered; women with diagnosed UF were more likely to have diagnosed depression (HR: 1.12 ; $95 \%$ CI 1.10 to 1.13 ), anxiety (HR: $1.11,95 \%$ CI 1.10 to 1.13 ) and self-directed violence (HR: $1.36,95 \%$ CI 1.21 to 1.52 ) than women without diagnosed UF. The magnitude of association was not appreciably altered when alternative definitions of the outcomes (online supplemental 


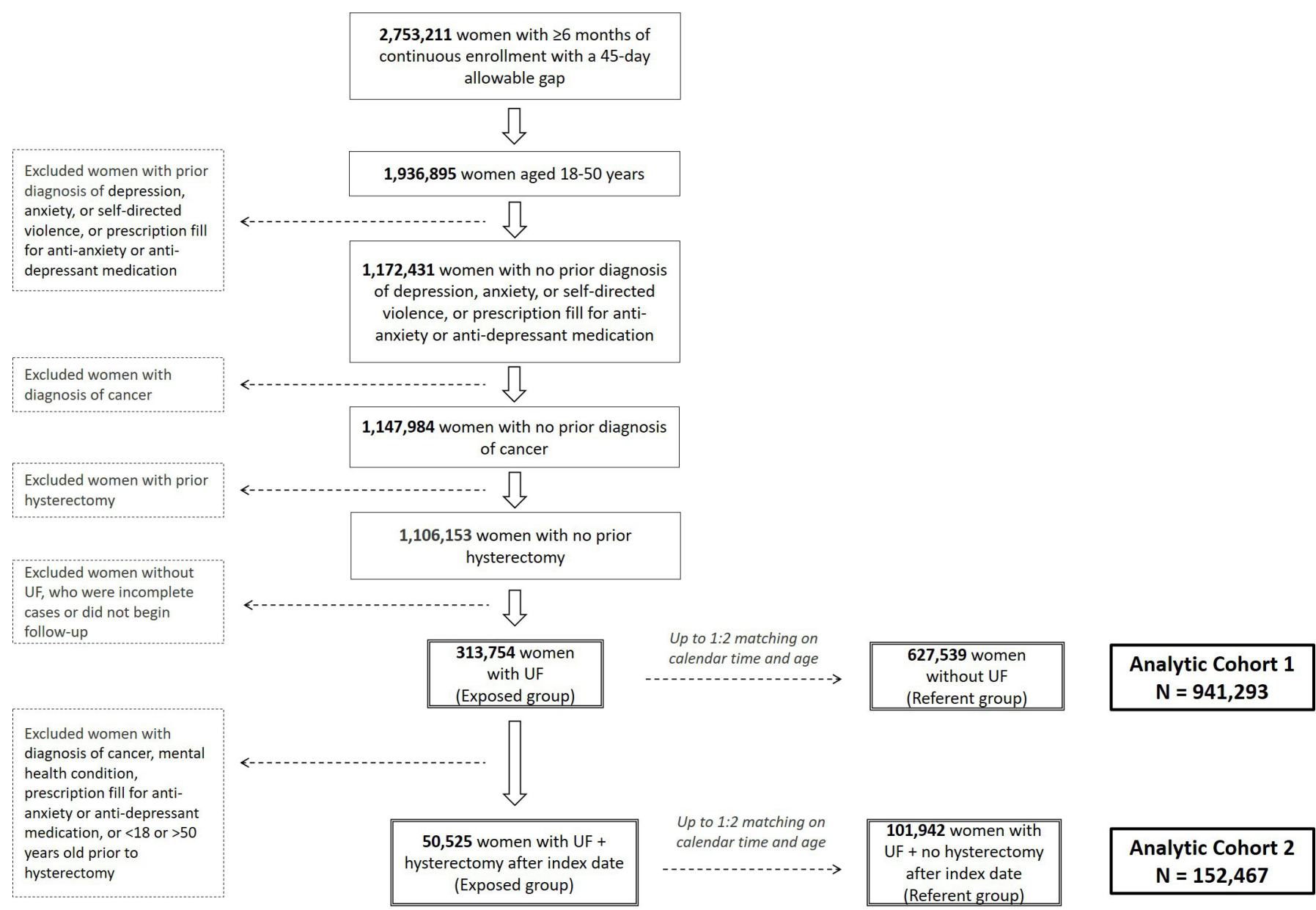

Figure 1 Cohort selection for women with uterine fibroids (UF) and a referent population of women without UF. Two study populations were used for the analysis. Analytical cohort 1 was used in the analyses to compare risk of mental health conditions in women with and without UF. Cohort entry date for analytical cohort 1 was the date of the second claim or the date of the UF claim following the ultrasonography/MRI, whichever came first, for the exposed group (UF diagnosis) and the date of a general medical or annual gynaecological examination for the referent group (no UF diagnosis). Analytical cohort 2 was used in the analyses to compare the risk of mental health conditions in women with UF who had a hysterectomy to women with UF who did not have a hysterectomy. Cohort entry date for analytical cohort 2 was the date of the hysterectomy after a diagnosis of UF for the exposed group and the date of a general medical or annual gynaecological examination after the diagnosis of UF for the referent group.

table 4) or exposure group (online supplemental table 5) were used.

The magnitude of association between diagnosed UF and diagnosed mental health outcomes was lowest in Black women and greatest in Asian women (table 2). The association between diagnosed UF and mental health outcomes was strongest among women with diagnosed UF and pain symptoms, with or without HMB (table 3). The magnitude of association between diagnosed UF and each mental health outcome was consistent across all age categories (online supplemental table 6) and the incidence rates and measures of association by decade of cohort entry were not meaningfully different (online supplemental table 7).

During the follow-up, 63523 (20.2\%) women with UF had a hysterectomy. Of those, 50525 women were matched to 101942 who did not have a hysterectomy (figure 1). Median follow-up time after hysterectomy was 620 days (IQR: 238, 1351) for depression, 611 days (IQR: 235, 1327) for anxiety and 673 days (IQR: 258, 1475) for self-directed violence. Hysterectomy was associated with a $22 \%$ higher rate of depression, $13 \%$ higher rate of anxiety and $86 \%$ higher rate of self-directed violence, after adjusting for covariates and prior procedures to treat UF (table 4). When the referent group was not censored on hysterectomy, the magnitude of the association was attenuated but remained significant. The HR comparing women who underwent a hysterectomy on the index date to women who did not was 1.09 (95\% CI 1.05 to 1.12) for depression, 1.06 (95\% CI 1.03 to 1.09$)$ for anxiety and 1.43 (95\% CI 1.12 to 1.82$)$ for self-directed violence.

\section{DISCUSSION}

Among women without prior diagnoses of anxiety, depression or self-harm, those with newly diagnosed UF are more likely to be diagnosed or treated for anxiety, depression and self-harm compared with women without diagnosed UF. These associations were highest among women experiencing pain-related UF symptoms. The magnitude of association varied by race and was lowest among non-Hispanic Black women. Women with diagnosed UF had a greater risk of self-directed harm, although the absolute rate was low (6 per 10000 person-years).

\section{Clinical context to prior studies}

A cohort study conducted in an administrative database in Taiwan reported increased rates of depression in women 
Table 1 Baseline characteristics in women with diagnosed uterine fibroids (UF) and women without diagnosed UF

\begin{tabular}{|c|c|c|c|}
\hline Variable & Diagnosed UF & No diagnosed UF* & Difference $(95 \% \mathrm{CI})$ between groups \\
\hline $\mathrm{n}$ & 313754 & 627539 & \\
\hline \multicolumn{4}{|l|}{ Demographics } \\
\hline Age, mean (SD) & $40.3(6.5)$ & $40.7(6.5)$ & $-0.4(-0.4$ to -0.3$)$ \\
\hline \multicolumn{4}{|l|}{ Race, n (\%) } \\
\hline White & $168479(53.7)$ & $429656(68.5)$ & $-14.8 \%(-15.0 \%$ to $-14.6 \%)$ \\
\hline Asian & $23776(7.6)$ & $50586(8.1)$ & $-0.5 \%(-0.6 \%$ to $-0.4 \%)$ \\
\hline Black & $74601(23.8)$ & $66365(10.6)$ & $13.2 \%(13.0 \%$ to $13.4 \%)$ \\
\hline Hispanic & $46898(14.9)$ & $80932(12.9)$ & $2.1 \%(1.9 \%$ to $2.2 \%)$ \\
\hline \multicolumn{4}{|l|}{ Region of residence, $n$ (\%) } \\
\hline Northeast & $41689(13.3)$ & $76374(12.2)$ & $1.1 \%(1.0 \%$ to $1.3 \%)$ \\
\hline Midwest & $60629(19.3)$ & $153106(24.4)$ & $-5.1 \%(-5.2 \%$ to $-4.9 \%)$ \\
\hline South & $159774(50.9)$ & $282796(45.1)$ & $5.9 \%(5.6 \%$ to $6.1 \%)$ \\
\hline West & $51662(16.5)$ & $115263(18.4)$ & $-1.9 \%(-2.1 \%$ to $-1.7 \%)$ \\
\hline Encounter with a mental health provider & $12073(3.8 \%)$ & $26678(4.3 \%)$ & $-0.4 \%(-0.5 \%$ to $-0.3 \%)$ \\
\hline \multicolumn{4}{|l|}{ Symptoms, n (\%) } \\
\hline Heavy menstrual bleeding & $134103(42.7)$ & $77223(12.3)$ & $30.4 \%(30.2 \%$ to $30.6 \%)$ \\
\hline Anaemia or blood transfusion & $18459(5.9)$ & $9661(1.5)$ & $4.3 \%(4.3 \%$ to $4.4 \%)$ \\
\hline Dysmenorrhoea & $36067(11.5)$ & $22187(3.5)$ & $8.0 \%(7.8 \%$ to $8.1 \%)$ \\
\hline Dyspareunia & $6768(2.2)$ & $6367(1.0)$ & $1.1 \%(1.1 \%$ to $1.2 \%)$ \\
\hline Pelvic pain & $107601(34.3)$ & $118774(18.9)$ & $15.4 \%(15.2 \%$ to $15.6 \%)$ \\
\hline \multicolumn{4}{|l|}{ Medical conditions, n (\%) } \\
\hline Pregnancy & $44055(14.0)$ & 93769 (14.9) & $-0.9 \%(-1.1 \%$ to $-0.8 \%)$ \\
\hline Live birth & $21174(6.7)$ & $70697(11.3)$ & $-4.5 \%(-4.6 \%$ to $-4.4 \%)$ \\
\hline Infertility & $70172(22.4)$ & $90879(14.5)$ & $7.9 \%(7.7 \%$ to $8.1 \%)$ \\
\hline Allergic rhinitis & $49945(15.9)$ & $112218(17.9)$ & $-2.0 \%(-2.1 \%$ to $-1.8 \%)$ \\
\hline Asthma & $22044(7.0)$ & $41509(6.6)$ & $0.4 \%(0.3 \%$ to $0.5 \%)$ \\
\hline Irritable bowel syndrome & $6520(2.1)$ & $12850(2.0)$ & $0.0 \%(0.0 \%$ to $0.1 \%)$ \\
\hline Psoriasis & $2582(0.8)$ & $7093(1.1)$ & $-0.3 \%(-0.3 \%$ to $-0.3 \%)$ \\
\hline Eczema & $26217(8.4)$ & $63725(10.2)$ & $-1.8 \%(-1.9 \%$ to $-1.7 \%)$ \\
\hline Chronic low back pain & $57187(18.2)$ & $118266(18.8)$ & $-0.6 \%(-0.8 \%$ to $-0.5 \%)$ \\
\hline Fatigue & $63534(20.2)$ & $122462(19.5)$ & $0.7 \%(0.6 \%$ to $0.9 \%)$ \\
\hline Vitamin D deficiency & $12289(3.9)$ & $25007(4.0)$ & $0.1 \%(-0.2 \%$ to $0.0 \%)$ \\
\hline Chronic headaches/migraines & $45976(14.7)$ & $89816(14.3)$ & $0.3 \%(0.2 \%$ to $0.5 \%)$ \\
\hline Thyroid disease & $38741(12.3)$ & $77470(12.3)$ & $0.0 \%(-0.1 \%$ to $0.1 \%)$ \\
\hline Hypothyroidism & $31080(9.9)$ & $66442(10.6)$ & $-0.7 \%(-0.8 \%$ to $-0.6 \%)$ \\
\hline Type 2 diabetes mellitus & $17018(5.4)$ & $26010(4.1)$ & $1.3 \%(1.2 \%$ to $1.4 \%)$ \\
\hline Hyperlipidaemia & $57158(18.2)$ & $115477(18.4)$ & $-0.2 \%(-0.3 \%$ to $0.0 \%)$ \\
\hline Hypertension & $51029(16.3)$ & $77184(12.3)$ & $4.0 \%(3.8 \%$ to $4.1 \%)$ \\
\hline Fibromyalgia & $15213(4.8)$ & $33244(5.3)$ & $-0.4 \%(-0.5 \%$ to $-0.4 \%)$ \\
\hline \multicolumn{4}{|l|}{ Medication use, $n$ (\%) } \\
\hline Combined hormonal contraceptives & $68719(21.9)$ & $169948(27.1)$ & $-5.2 \%(-5.4 \%$ to $-5.0 \%)$ \\
\hline Oral progestin & $9262(3.0)$ & $29120(4.6)$ & $-1.7 \%(-1.8 \%$ to $-1.6 \%)$ \\
\hline Depot medroxyprogesterone acetate & $18356(5.9)$ & $24834(4.0)$ & $1.9 \%(1.8 \%$ to $2.0 \%)$ \\
\hline Intrauterine device & $21921(7.0)$ & $59541(9.5)$ & $-2.5 \%(-2.6 \%$ to $-2.4 \%)$ \\
\hline Non-opioid analgesics & $85557(27.3)$ & $149745(23.9)$ & $3.4 \%(3.2 \%$ to $3.6 \%)$ \\
\hline Opioid analgesic & $107983(34.4)$ & $203783(32.5)$ & $1.9 \%(1.7 \%$ to $2.1 \%)$ \\
\hline Antihypertensives & $41595(13.3)$ & $68295(10.9)$ & $2.4 \%(2.2 \%$ to $2.5 \%)$ \\
\hline Statins & $11546(3.7)$ & 24597 (3.9) & $-0.2 \%(-0.3 \%$ to $-0.2 \%)$ \\
\hline Corticosteroids & $89124(28.4)$ & 199403 (31.8) & $-3.4 \%(-3.6 \%$ to $-3.2 \%)$ \\
\hline Tranexamic acid & $1821(0.6)$ & $809(0.1)$ & $0.5 \%(0.4 \%$ to $0.5 \%)$ \\
\hline \multicolumn{4}{|l|}{ Prior procedures, n (\%) } \\
\hline Uterine artery embolisation & $36(0.0)$ & $5(0.0)$ & $0.0 \%(0.0 \%$ to $0.0 \%)$ \\
\hline Myomectomy & $6612(2.1)$ & $0(0.0)$ & $2.1 \%(2.1 \%$ to $2.2 \%)$ \\
\hline
\end{tabular}

* Matched referent includes women with no UF or prior myomectomy. 
Table 2 Association between UF and diagnosis of mental health outcomes, overall and stratified by race/ethnicity

\begin{tabular}{|c|c|c|c|c|c|c|}
\hline & \multicolumn{2}{|l|}{ Depression } & \multicolumn{2}{|l|}{ Anxiety } & \multicolumn{2}{|c|}{ Self-directed violence } \\
\hline & $\begin{array}{l}\text { Diagnosed } \\
\text { UF }\end{array}$ & $\begin{array}{l}\text { No diagnosed } \\
\text { UF }\end{array}$ & $\begin{array}{l}\text { Diagnosed } \\
\text { UF }\end{array}$ & $\begin{array}{l}\text { No diagnosed } \\
\text { UF }\end{array}$ & $\begin{array}{l}\text { Diagnosed } \\
\text { UF }\end{array}$ & $\begin{array}{l}\text { No diagnosed } \\
\text { UF }\end{array}$ \\
\hline \multicolumn{7}{|l|}{ Overall } \\
\hline $\mathrm{n}$ & 313754 & 627539 & 313754 & 627539 & 313754 & 627539 \\
\hline Events, $\mathrm{n}$ & 26171 & 46103 & 34003 & 60683 & 509 & 616 \\
\hline $\begin{array}{l}\text { Days between index date } \\
\text { and outcome diagnosis, } \\
\text { median (IQR) }\end{array}$ & $609(240,1330)$ & $607(234,1313)$ & $596(234,1302)$ & $596(229,1289)$ & $670(264,1471)$ & $662(256,1432)$ \\
\hline Rate per 1000 person-years & 30.9 & 27.8 & 41.0 & 37.3 & 0.6 & 0.3 \\
\hline Age-matched HR (95\% Cl) & 1.12 (1.10 to 1.13$)$ & 1.0 (Ref) & 1.10 (1.09 to 1.12 ) & 1.0 (Ref) & 1.60 (1.42 to 1.80$)$ & 1.0 (Ref) \\
\hline Adjusted HR $(95 \% \mathrm{Cl})^{*}$ & 1.12 (1.10 to 1.13$)$ & 1.0 (Ref) & 1.12 (1.10 to 1.13$)$ & 1.0 (Ref) & 1.46 (1.29 to 1.64$)$ & 1.0 (Ref) \\
\hline \multicolumn{7}{|l|}{ Non-Hispanic White } \\
\hline $\mathrm{n}$ & 168479 & 429656 & 168479 & 429656 & 168479 & 429656 \\
\hline Events, $\mathrm{n}$ & 15888 & 34612 & 20240 & 44905 & 263 & 430 \\
\hline Rate per 1000 person-years & 34.1 & 29.6 & 44.4 & 39.1 & 0.5 & 0.3 \\
\hline Age-matched HR $(95 \% \mathrm{Cl})$ & 1.16 (1.14 to 1.18$)$ & 1.0 (Ref) & 1.14 (1.12 to 1.15$)$ & 1.0 (Ref) & 1.51 (1.29 to 1.76$)$ & 1.0 (Ref) \\
\hline Adjusted HR $(95 \% \mathrm{Cl})^{*}$ & $1.13(1.10$ to 1.15$)$ & 1.0 (Ref) & $1.12(1.10$ to 1.14$)$ & 1.0 (Ref) & $1.41(1.21$ to 1.65$)$ & 1.0 (Ref) \\
\hline \multicolumn{7}{|l|}{ Non-Hispanic Black } \\
\hline $\mathrm{n}$ & 74601 & 66365 & 74601 & 66365 & 74601 & 66365 \\
\hline Events, $\mathrm{n}$ & 5465 & 4383 & 6937 & 5479 & 141 & 81 \\
\hline Rate per 1000 person-years & 27.5 & 26.8 & 35.5 & 33.9 & 0.7 & 0.5 \\
\hline Age-matched HR $(95 \% \mathrm{Cl})$ & 1.04 (1.00 to 1.08$)$ & 1.0 (Ref) & 1.05 (1.02 to 1.09$)$ & 1.0 (Ref) & 1.43 (1.09 to 1.88$)$ & 1.0 (Ref) \\
\hline Adjusted HR $(95 \% \mathrm{Cl})^{*}$ & $1.03(0.99$ to 1.07$)$ & 1.0 (Ref) & 1.05 (1.02 to 1.09$)$ & 1.0 (Ref) & 1.38 (1.05 to 1.82$)$ & 1.0 (Ref) \\
\hline \multicolumn{7}{|l|}{ Hispanic } \\
\hline $\mathrm{n}$ & 46898 & 80932 & 46898 & 80932 & 46898 & 80932 \\
\hline Events, $\mathrm{n}$ & 3821 & 5390 & 5230 & 7653 & 78 & 79 \\
\hline Rate per 1000 person-years & 32.4 & 27.8 & 45.5 & 40.5 & 0.6 & 0.4 \\
\hline Age-matched HR $(95 \% \mathrm{Cl})$ & 1.17 (1.13 to 1.22$)$ & 1.0 (Ref) & 1.13 (1.09 to 1.17 ) & 1.0 (Ref) & 1.60 (1.17 to 2.19$)$ & 1.0 (Ref) \\
\hline Adjusted HR $(95 \% \mathrm{Cl})^{*}$ & $1.15(1.10$ to 1.20$)$ & 1.0 (Ref) & $1.11(1.08$ to 1.15$)$ & 1.0 (Ref) & 1.57 (1.14 to 2.15$)$ & 1.0 (Ref) \\
\hline \multicolumn{7}{|l|}{ Asian } \\
\hline $\mathrm{n}$ & 23776 & 50586 & 23776 & 50586 & 23776 & 50586 \\
\hline Events, $\mathrm{n}$ & 997 & 1718 & 1596 & 2646 & 27 & 26 \\
\hline Rate per 1000 person-years & 15.6 & 13.2 & 25.5 & 20.6 & 0.4 & 0.2 \\
\hline Age-matched HR $(95 \% \mathrm{Cl})$ & $1.19(1.10$ to 1.29$)$ & 1.0 (Ref) & 1.24 (1.17 to 1.32$)$ & 1.0 (Ref) & 2.09 (1.22 to 3.57$)$ & 1.0 (Ref) \\
\hline Adjusted HR $(95 \% \mathrm{Cl})^{*}$ & 1.17 (1.08 to 1.27$)$ & 1.0 (Ref) & $1.23(1.16$ to 1.31$)$ & 1.0 (Ref) & 1.98 (1.15 to 3.41$)$ & 1.0 (Ref) \\
\hline
\end{tabular}

*HR adjusted for race, region of residence, allergic rhinitis, asthma, irritable bowel syndrome (IBS), eczema, chronic low back pain, fatigue, vitamin D deficiency, chronic headaches/migraines, thyroid disease, hypothyroidism, type 2 diabetes mellitus, hyperlipidaemia, hypertension, fibromyalgia, live birth and use of antihypertensives, corticosteroids, non-opioid analgesics, opioid analgesics, combined hormonal contraceptives, statins, oral progestin, depot medroxyprogesterone acetate or progestogen-releasing intrauterine system. Race-stratified models adjusted for all the covariates listed above except race.

UF, uterine fibroid.

with an ICD diagnosis code for UF (HR: 1.46, 95\% CI 1.36 to 1.57$)$ compared with women without. ${ }^{8}$ Our results suggest that UF is also associated with diagnosis of anxiety and self-directed violence. UF and mental health disorders are chronic conditions with significant delay in clinical diagnosis after the initial presentation of symptoms. Therefore, temporality in relation to disease onset is hard to establish from administrative claims data. In the Black Women's Health Study, women with diagnosed depression had a relative risk for incident self-reported UF of 1.25 (95\% CI 1.13 to 1.39 ) compared with women without depression. ${ }^{5}$ Thus, the relation of UF and mental health disorders may be bidirectional, and an underlying common cause of UF and depression, such as an abnormal hormonal milieu, may increase a woman's susceptibility to both diseases.

\section{Pain-related symptoms and mental health}

The association between diagnosed UF and diagnosed mental health outcomes was stronger among women with pain-related symptoms, implying that poorly managed pain is an important pathway underlying the association between UF and subsequent mental health outcomes. Chronic pain and depression may share common neuroplasticity mechanisms, including effects on monoamine neurotransmitters, brain-derived neurotrophic factor and glutamate and its receptor subtypes. ${ }^{13}$ Further, the prevalence of anxiety and depression is high in patients with chronic pain conditions, including chronic pelvic pain, ${ }^{14}$ low back pain ${ }^{15}$ and migraines. ${ }^{16}$

\section{Hysterectomy and depression}

Among women with diagnosed UF, hysterectomy was associated with greater rates of diagnosed mental health outcomes. Prior 
Table 3 Association between UF and diagnosis of mental health outcomes, stratified by symptom-based UF phenotypes

\begin{tabular}{|c|c|c|c|c|c|}
\hline & \multirow[b]{2}{*}{ No diagnosed UF } & \multicolumn{4}{|c|}{ Diagnosed UF phenotype } \\
\hline & & No bleeding or pain & Heavy bleeding only* & Pain only† & Bleeding and pain \\
\hline$n$ & 627539 & 110259 & 100001 & 86650 & 95889 \\
\hline Events, $\mathrm{n}$ & 46103 & 8352 & 8158 & 7230 & 8331 \\
\hline Rate per 1000 person-years & 27.8 & 26.6 & 28.6 & 32.3 & 33.1 \\
\hline Age-matched HR $(95 \% \mathrm{Cl})$ & 1.0 (Ref) & 0.95 (0.93 to 0.98$)$ & 1.08 (1.05 to 1.11$)$ & $1.18(1.15$ to 1.21$)$ & $1.26(1.23$ to 1.30$)$ \\
\hline \multicolumn{6}{|l|}{ Anxiety } \\
\hline Events, $\mathrm{n}$ & 60683 & 11011 & 10257 & 9868 & 11106 \\
\hline Rate per 1000 person-years & 37.3 & 35.9 & 36.6 & 45.3 & 45.2 \\
\hline Age-matched HR $(95 \% \mathrm{Cl})$ & 1.0 (Ref) & 0.97 (0.95 to 0.99$)$ & 1.03 (1.01 to 1.06$)$ & 1.18 (1.15 to 1.21$)$ & 1.21 (1.19 to 1.24$)$ \\
\hline Adjusted HR $(95 \% \mathrm{Cl}) \ddagger$ & 1.0 (Ref) & $1.03(1.00$ to 1.05$)$ & 1.06 (1.04 to 1.09$)$ & $1.17(1.14$ to 1.20$)$ & $1.18(1.15$ to 1.21$)$ \\
\hline Age-matched HR $(95 \% \mathrm{Cl})$ & 1.0 (Ref) & $1.04(0.84$ to 1.30$)$ & $1.29(1.04$ to 1.59$)$ & $1.86(1.50$ to 2.31$)$ & 1.87 (1.52 to 2.29$)$ \\
\hline Adjusted HR $(95 \% \mathrm{Cl}) \ddagger$ & 1.0 (Ref) & $0.98(0.78$ to 1.23$)$ & 1.16 (0.93 to 1.44$)$ & 1.66 (1.33 to 2.08$)$ & 1.68 (1.35 to 2.09$)$ \\
\hline
\end{tabular}

*Heavy bleeding defined as heavy menstrual bleeding (menorrhagia or menometrorrhagia), anaemia or blood transfusion.

tPain defined as dysmenorrhoea, dyspareunia or pelvic pain.

¥HR adjusted for race, region of residence, allergic rhinitis, asthma, irritable bowel syndrome (IBS), eczema, chronic low back pain, fatigue, vitamin D deficiency, chronic headaches/migraines, thyroid disease, hypothyroidism, type 2 diabetes mellitus, hyperlipidaemia, hypertension, fibromyalgia, live birth and use of antihypertensives, corticosteroids, non-opioid analgesics, opioid analgesics, combined hormonal contraceptives, statins, oral progestin, depot medroxyprogesterone acetate or progestogen-releasing intrauterine system.

UF, uterine fibroid.

studies reported improvement in quality of life and psychological conditions after a hysterectomy, ${ }^{17-19}$ although these studies were limited by short follow-up, small sample size or outcomes reported around the time of surgery. Recent cohort studies found a 20\%-78\% higher risk of depression ${ }^{20-22}$ and a $22 \%$ higher risk of anxiety ${ }^{20}$ after a hysterectomy for any benign condition, and the risk was greatest in women experiencing perioperative pain. ${ }^{23}$ When stratified by indication, hysterectomy for UF was associated with an HR of 1.12 (95\% CI 0.92 to 1.35) for depression and 1.14 (95\% CI 0.93 to 1.40$)$ for anxiety. ${ }^{20}$ Although the CIs were wide, due to a low number of cases $(n=223$ for depression and 199 for anxiety), the point estimates were consistent with results from the current study. Conversely, in a Taiwan administrative database, hysterectomy for UF was associated with a $36 \%$ lower risk of diagnosed depression (HR: 0.64; $95 \%$ CI 0.51 to 0.81$)^{8}$; however, the overall rate of diagnosed depression (7.5 per 1000 person-years) was half the rate of depression in Asian women in the present study. Discrepancies may be due to reporting bias, differences in healthcare practice or underlying representation of the administrative databases. While we adjusted for myomectomy and UAE procedures prior to hysterectomy, we are not able to control completely for UF severity that may influence the decision to undergo hysterectomy.

Hysterectomy may lead to depression through numerous pathways, including oestrogen deficiency caused by oophorectomy $^{24}$ if ovaries are removed, or loss of endocrine activity of the endometrium, ${ }^{25}$ or detrimental effects of the ovaries via ovarian-independent mechanisms, in the context of ovarian conservation. ${ }^{26}$ The procedure codes for hysterectomy and/or oophorectomy limited the ability to separate hysterectomy with ovarian conservation and ovarian removal in the analysis. Additionally, the loss of fertility in women before the end of childbearing may influence future mental health. ${ }^{27}$ However, data on pregnancy intentions and desired family size were unavailable.

\section{Racial disparities in UF and mental illness}

Black women are diagnosed with UF at earlier ages, experience greater symptom severity and are more likely to have hysterectomy for UF. ${ }^{28}$ Further, these surgeries are more likely to be performed abdominally rather than laparoscopically. ${ }^{29}$ Despite greater disease burden, rates of diagnosed depression and anxiety were not elevated in Black women. Individuals from minority groups are less likely to meet criteria for depression and anxiety disorders or receive psychotropic medications compared with White women. ${ }^{30}$ In addition, the stigma of mental health conditions may lead to self-silencing of symptoms and underdiagnosis of mental illness. ${ }^{31}$ The lower rates of diagnosed depression in Black women with UF mirror the 'Black-White depression paradox', in which Black women have greater exposure to psychological, social and economic stressors relative to White women, yet the prevalence of diagnosed depression is lower. ${ }^{32}$ Additional research is needed to understand whether these observed differences are due to differences in disease burden, clinical or cultural presentation of symptoms and/or access to healthcare.

\section{Postulated biological mechanisms}

There are several plausible biological pathways that may link UF and depression. First, hormonal dysregulation may be a common cause of both depression and UF. ${ }^{33}$ Sex steroid hormones may influence the pathogenesis of $\mathrm{UF}^{34}$ and the serum levels of sex hormones are different in women with depression relative to women without. ${ }^{35}$ Psychological stressors, such as depression, are associated with dysregulation of the hypothalamic-pituitaryadrenal (HPA) axis and these HPA abnormalities may influence the hypothalamic-pituitary-ovarian axis and alter sex steroid hormone production. ${ }^{36}$ Other psychosocial stressors, such as childhood abuse and perceived racism, have been associated 
Table 4 Association between hysterectomy after UF diagnosis and diagnosis of mental health outcomes, restricted to women with diagnosed UF

\begin{tabular}{|c|c|c|}
\hline & Hysterectomy & No hysterectomy \\
\hline $\mathrm{n}$ & 50525 & 101942 \\
\hline \multicolumn{3}{|l|}{ Depression } \\
\hline Events, $\mathrm{n}$ & 4368 & 6588 \\
\hline $\begin{array}{l}\text { Days between index date and } \\
\text { outcome diagnosis, median (IQR) }\end{array}$ & $638(247,1394)$ & $612(235,1330)$ \\
\hline Rate per 1000 person-years & 31.1 & 24.3 \\
\hline Age-matched HR (95\% Cl) & 1.29 (1.24 to 1.34$)$ & 1.0 (Ref) \\
\hline Adjusted HR $(95 \% \mathrm{Cl})^{*}$ & 1.22 (1.17 to 1.27$)$ & 1.0 (Ref) \\
\hline \multicolumn{3}{|l|}{ Anxiety } \\
\hline Events, $\mathrm{n}$ & 5259 & 8970 \\
\hline $\begin{array}{l}\text { Days between index date and } \\
\text { outcome diagnosis, median (IQR) }\end{array}$ & $631(245,1378)$ & $601(231,1302)$ \\
\hline Rate per 1000 person-years & 38.0 & 33.7 \\
\hline Age-matched HR (95\% Cl) & 1.13 (1.09 to 1.17$)$ & 1.0 (Ref) \\
\hline Adjusted HR $(95 \% \mathrm{Cl})^{*}$ & 1.13 (1.09 to 1.17$)$ & 1.0 (Ref) \\
\hline \multicolumn{3}{|l|}{ Self-directed violence } \\
\hline Events, $\mathrm{n}$ & 99 & 97 \\
\hline $\begin{array}{l}\text { Days between index date and } \\
\text { outcome diagnosis, median (IQR) }\end{array}$ & $704(271,1546)$ & $657(253,1435)$ \\
\hline Rate per 1000 person-years & 0.7 & 0.3 \\
\hline Age-matched HR $(95 \% \mathrm{Cl})$ & 1.94 (1.47 to 2.57$)$ & 1.0 (Ref) \\
\hline Adjusted HR $(95 \% \mathrm{Cl})^{*}$ & 1.86 (1.39 to 2.49$)$ & 1.0 (Ref) \\
\hline
\end{tabular}

*HR adjusted for race, region of residence, allergic rhinitis, asthma, irritable bowel syndrome (IBS), eczema, chronic low back pain, fatigue, vitamin D deficiency, chronic headaches/migraines, thyroid disease, hypothyroidism, type 2 diabetes mellitus, hyperlipidaemia, hypertension, fibromyalgia, live birth and use of antihypertensives, corticosteroids, non-opioid analgesics, opioid analgesics, combined hormonal contraceptives, statins, oral progestin, depot medroxyprogesterone acetate, progestogenreleasing intrauterine system, prior myomectomy and prior uterine artery embolisation (UAE)

UF, uterine fibroid.

with UF. ${ }^{37} 38$ Pain symptoms may also be chronic stressors that lead to depression and anxiety in women with symptomatic UF.

\section{Strengths and limitations}

Strengths of this study included the use of a large database with a contemporary cohort of commercially insured individuals with broad geographic coverage. All patients were enrolled in an insurance plan and had access to healthcare and mental healthcare services, and the women in the unexposed group were required to have at least one healthcare encounter to minimise bias in healthseeking behaviours between patients with and without diagnosed UF. Finally, the prospective design of this study and exclusion of prevalent mental health diagnoses allow for the establishment of the temporal sequence between the diagnosis of UF and mental health outcomes.

There are limitations of the study that warrant discussion. First, misclassification of the exposure is likely, given that a significant proportion of women with UF are asymptomatic ${ }^{2}$ and may not have claims with UF diagnosis codes. Due to this misclassification the associations may be overestimated and may be most generalisable to women with symptomatic UF. The optimal interpretation of these results is that women seeking care for UF are at greater risk of receiving a diagnosis of mood disorders.

The diagnosis of mental health conditions was likely underestimated, as only women who sought medical attention for these conditions would be identified through claims. Although all women were enrolled in a commercial insurance plan, the access to mental healthcare and healthcare in general may not be equal for everyone. We are unable to assess subsyndromal symptoms of depression and anxiety in this secondary database; only diagnosed disease was captured. Additionally, UF and mood disorders are chronic conditions, and due to the limited longitudinal observability in individuals in the claims data, we were unable to assess the timing of the onset of either condition. Women who had undiagnosed UF at the time of a mental health diagnosis did not contribute to the analysis. As these women may have greater disease burden, their exclusion from the analysis may have underestimated the impact of symptomatic UF on risk of mood disorders. Importantly, this study assessed the temporal relationship of receiving a clinical diagnosis of mood disorders in women after a diagnosis of UF.

Race, which was imputed in the majority of the population, ${ }^{12}$ is misclassified in these data. The imputation method was validated previously and demonstrated $97 \%$ specificity, and $71 \%$ positive predictive value, but only $48 \%$ sensitivity, for estimating the race of Black individuals. ${ }^{39}$ This misclassification would increase the heterogeneity within racial subgroups and underestimate any differences in the association between UF and diagnosed mental health conditions between these subgroups. Finally, the generalisability of these results to an uninsured population may be limited.

\section{CONCLUSIONS}

Women with diagnosed UF may be at a higher risk for diagnosed depression, anxiety and self-directed violence relative to women without, particularly among women who experience pain symptoms. Obstetricians and gynaecologists can play an important role in early detection, prevention and treatment of mood disorders by screening for symptoms at routine examinations. ${ }^{40}$ The potential for precipitating or exacerbating mental health disorders may be considered while counselling patients on UF treatment options, especially hysterectomy. A multidisciplinary approach that identifies women with UF at higher risk of depression and anxiety may improve management of mood disorders and improve strategies for prevention.

\section{What is already known on this subject}

- The prevalence of depression and anxiety is high among women with uterine fibroids (UF). The rate of diagnosed mental health outcomes among women with a diagnosed UF has not been well studied.

\section{What this study adds}

- Women with diagnosed UF, particularly those who experienced pain-related symptoms from UF or who underwent hysterectomy, are at higher risk for diagnosis of mental health outcomes than women without diagnosed UF. Obstetricians and gynaecologists can play an important role in early detection, prevention and treatment of mood disorders by screening for symptoms at routine examinations.

\section{Twitter Lauren A Wise @laurenannewise}

Contributors SEC generated the idea for the study, formulated an analytical plan, interpreted the results and drafted the manuscript. $\mathrm{CH}$ contributed to the design of the study, analysed the data, interpreted the results and revised the manuscript. $\mathrm{NPI}, \mathrm{CO}, \mathrm{WK}$ and LAW contributed to the design of the study and interpretation of 
the results, and revised the manuscript. All authors approved the final version of the manuscript.

Funding This work was funded by AbbVie, and Aetion received funding for conducting this study.

Disclaimer AbbVie participated in the study design, research, data collection, analysis and interpretation of data, writing, reviewing and approving the publication.

Competing interests $\mathrm{SEC}, \mathrm{CH}$ and $\mathrm{CO}$ are employees of AbbVie receiving stock and/or stock options. NPI was an employee of Aetion at the time the work was conducted and may hold stock options at Aetion. WK and LAW conducted this work as paid consultants to AbbVie but did not receive payment for authorship.

Patient consent for publication Not required.

Ethics approval This study was exempt from Institutional Review Board approval because it was a retrospective analysis of deidentified data.

Provenance and peer review Not commissioned; externally peer reviewed.

Data availability statement Data may be obtained from a third party and are not publicly available. This study used data from a commercially licensed database the Optum Clinformatics DataMart (OptumInsight, Eden Prairie, Minnesota). More information about data access can be found at www.optum.com/life-sciencessolutions.

Supplemental material This content has been supplied by the author(s). It has not been vetted by BMJ Publishing Group Limited (BMJ) and may not have been peer-reviewed. Any opinions or recommendations discussed are solely those of the author(s) and are not endorsed by BMJ. BMJ disclaims all liability and responsibility arising from any reliance placed on the content. Where the content includes any translated material, BMJ does not warrant the accuracy and reliability of the translations (including but not limited to local regulations, clinical guidelines, terminology, drug names and drug dosages), and is not responsible for any error and/or omissions arising from translation and adaptation or otherwise.

Open access This is an open access article distributed in accordance with the Creative Commons Attribution Non Commercial (CC BY-NC 4.0) license, which permits others to distribute, remix, adapt, build upon this work non-commercially, and license their derivative works on different terms, provided the original work is properly cited, appropriate credit is given, any changes made indicated, and the use is non-commercial. See: http://creativecommons.org/licenses/by-nc/4.0/.

\section{ORCID iDs}

Stephanie E Chiuve http://orcid.org/0000-0002-3524-8917

Lauren A Wise http://orcid.org/0000-0003-2138-3752

\section{REFERENCES}

1 Wright JD, Herzog TJ, Tsui J, et al. Nationwide trends in the performance of inpatient hysterectomy in the United States. Obstet Gynecol 2013;122:233-41.

2 Day Baird D, Dunson DB, Hill MC, et al. High cumulative incidence of uterine leiomyoma in black and white women: ultrasound evidence. Am J Obstet Gynecol 2003:188:100-7.

3 Khan AT, Shehmar M, Gupta JK. Uterine fibroids: current perspectives. Int J Womens Health 2014;6:95-114.

4 Ghant MS, Sengoba KS, Recht H, et al. Beyond the physical: a qualitative assessment of the burden of symptomatic uterine fibroids on women's emotional and psychosocial health. J Psychosom Res 2015;78:499-503.

5 Wise LA, Li S, Palmer JR, et al. Depressive symptoms and risk of uterine leiomyomata. Am J Obstet Gynecol 2015:212:617.e1-617.e10.

6 Gambadauro P, Carli V, Hadlaczky G. Depressive symptoms among women with endometriosis: a systematic review and meta-analysis. Am J Obstet Gynecol 2019;220:230-41

7 Berni TR, Morgan CL, Berni ER, et al. Polycystic ovary syndrome is associated with adverse mental health and neurodevelopmental outcomes. J Clin Endocrinol Metab 2018; 103:2116-25.

8 Shen T-C, Yang C-Y, Huang Y-J, et al. Risk of depression in patients with uterine leiomyoma: a nationwide population-based cohort study. J Affect Disord 2017:213:126-30.

9 Townsend L, Walkup JT, Crystal S, et al. A systematic review of validated methods for identifying depression using administrative data. Pharmacoepidemiol Drug Saf 2012;21 Suppl 1:163-73

10 Hedegaard H, Schoenbaum M, Claassen C, et al. Issues in developing a surveillance case definition for nonfatal suicide attempt and intentional self-harm using International classification of diseases, tenth revision, clinical modification (ICD-10CM) coded data. Natl Health Stat Report 2018:1-19.

11 Kessler RC, Berglund P, Borges G, et al. Trends in suicide ideation, plans, gestures, and attempts in the United States, 1990-1992 to 2001-2003. JAMA 2005;293:2487-95.
12 Jain A, Marshall J, Buikema A, et al. Autism occurrence by MMR vaccine status among US children with older siblings with and without autism. JAMA 2015:313:1534-40.

13 Sheng J, Liu S, Wang Y, et al. The link between depression and chronic pain: neural mechanisms in the brain. Neural Plast 2017:2017:9724371

14 Siqueira-Campos VME, Da Luz RA, de Deus JM, et al. Anxiety and depression in women with and without chronic pelvic pain: prevalence and associated factors. $J$ Pain Res 2019;12:1223-33.

15 Fernandez M, Colodro-Conde L, Hartvigsen J, et al. Chronic low back pain and the risk of depression or anxiety symptoms: insights from a longitudinal twin study. Spine J 2017; 17:905-12

16 McLean G, Mercer SW. Chronic migraine, comorbidity, and socioeconomic deprivation: cross-sectional analysis of a large nationally representative primary care database. $J$ Comorb 2017:7:89-95.

17 Darwish M, Atlantis E, Mohamed-Taysir T. Psychological outcomes after hysterectomy for benign conditions: a systematic review and meta-analysis. Eur J Obstet Gynecol Reprod Biol 2014;174:5-19.

18 Farquhar CM, Sadler L, Stewart AW. A prospective study of outcomes five years after hysterectomy in premenopausal women. Aust N Z J Obstet Gynaecol 2008:48:510-6.

19 Nicholson WK, Wegienka G, Zhang S, et al. Short-term health-related quality of life after hysterectomy compared with myomectomy for symptomatic leiomyomas. Obstet Gynecol 2019;134:261-9.

20 Laughlin-Tommaso SK, Satish A, Khan Z, et al. Long-term risk of de novo mental health conditions after hysterectomy with ovarian conservation: a cohort study. Menopause 2020;27:33-42.

21 Wilson L, Pandeya N, Byles J, et al. Hysterectomy and incidence of depressive symptoms in midlife women: the Australian Longitudinal Study on Women's Health. Epidemiol Psychiatr Sci 2018;27:381-92.

22 Harnod T, Chen W, Wang J-H, et al. Hysterectomies are associated with an increased risk of depression: a population-based cohort study. J Clin Med 2018;7:366.

23 Theunissen M, Peters ML, Schepers J, et al. Prevalence and predictors of depression and well-being after hysterectomy: an observational study. Eur J Obstet Gynecol Reprod Biol 2017;217:94-100

24 Rocca WA, Grossardt BR, Geda YE, et al. Long-term risk of depressive and anxiety symptoms after early bilateral oophorectomy. Menopause 2018;25:1275-85.

25 Koebele SV, Palmer JM, Hadder B, et al. Hysterectomy uniquely impacts spatial memory in a rat model: a role for the nonpregnant uterus in cognitive processes. Endocrinology 2019;160:1-19.

26 Stewart EA. Gonadotropins and the uterus: is there a gonad-independent pathway? Soc Gynecol Investig 2001;8:319-26.

27 Leppert PC, Legro RS, Kjerulff KH. Hysterectomy and loss of fertility: implications for women's mental health. J Psychosom Res 2007:63:269-74.

28 Stewart EA, Nicholson WK, Bradley L, et al. The burden of uterine fibroids for AfricanAmerican women: results of a national survey. J Womens Health 2013;22:807-16

29 Eltoukhi HM, Modi MN, Weston M, et al. The health disparities of uterine fibroid tumors for African American women: a public health issue. Am J Obstet Gynecol 2014:210:194-9.

30 Coleman KJ, Stewart C, Waitzfelder BE, et al. Racial-Ethnic differences in psychiatric diagnoses and treatment across 11 health care systems in the mental health research network. Psychiatr Serv 2016;67:749-57.

31 Abrams JA, Hill A, Maxwell M. Underneath the mask of the strong black woman schema: disentangling influences of strength and Self-Silencing on depressive symptoms among U.S. black women. Sex Roles 2019;80:517-26.

32 Barnes DM, Bates LM. Do racial patterns in psychological distress shed light on the Black-White depression paradox? A systematic review. Soc Psychiatry Psychiatr Epidemiol 2017;52:913-28.

33 Tang $H$, Zhang Y. Identification and bioinformatics analysis of overlapping differentially expressed genes in depression, papillary thyroid cancer and uterine fibroids. Exp Ther Med 2018;15:4810-6.

34 Rein MS, Barbieri RL, Friedman AJ. Progesterone: a critical role in the pathogenesis of uterine myomas. Am J Obstet Gynecol 1995;172:14-18.

35 Holsen LM, Lancaster K, Klibanski A, et al. HPA-axis hormone modulation of stress response circuitry activity in women with remitted major depression. Neuroscience 2013;250:733-42.

36 Breen KM, Billings HJ, Wagenmaker ER, et al. Endocrine basis for disruptive effects of cortisol on preovulatory events. Endocrinology 2005;146:en.2004-1457:2107-15.

37 Wise LA, Palmer JR, Rosenberg L. Lifetime abuse victimization and risk of uterine leiomyomata in black women. Am J Obstet Gynecol 2013;208:272.e1-272.e13.

38 Wise LA, Palmer JR, Cozier YC, et al. Perceived racial discrimination and risk of uterine leiomyomata. Epidemiology 2007;18:747-57.

39 DeFrank JT, Bowling JM, Rimer BK. Triangulating differential nonresponse by race in a telephone survey. Prev Chronic Dis 2007;4:A60

40 Bhat A, Reed SD, Unützer J. The Obstetrician-Gynecologist's role in detecting, preventing, and treating depression. Obstet Gynecol 2017;129:157-63. 